\title{
Skin flap squamous cell carcinoma developed after urethroplasty
}

\author{
Danilo Abate, Giuseppe Giusti, Nicola Caria, Giuseppe De Vita, Marco Lucci Chiarissi, \\ Antonello De Lisa \\ Clinica Urologica, Università degli Studi di Cagliari, Ospedale SS. Trinità, Cagliari, Italy.
}

\begin{abstract}
Summary Objectives: To describe our experience in diagnosis and treatment of urethral carcinoma following urethroplasty with a Orandi penile skin flap. Material and methods: Our patient underwent to Orandi penile skin flap urethroplasty then developed a urethral epidermoid carcinoma on the flap approximately 15 years later.

We treated this case with a partial penectomy surgery and perineostomy. Surgery was followed by chemotherapy with cisplatin and 5-fluorouracil. The progression of the disease led to a salvage surgery of total penectomy and asportation of testicles and scrotum.

Results: Despite the success of the surgery, the disease progressed and three months after the last surgical operation the patient died.

Conclusions: Urethral carcinoma on skin flap is a rare complication of the urethroplasty surgery but with severe consequences, so we recommend to undertake a long-term urological follow up in patients undergone such kind of surgery.
\end{abstract}

KEY WORDS: Skin flap; Squamous cell carcinoma; Urethroplasty.

Submitted 13 April 2018; Accepted 29 April 2018

\section{INTRODUCTION}

According to the American Association of Urology (AUA) guidelines, male urethral stenosis can be treated with urethral dilatation, endoscopic urethrotomy, and surgical urethroplasty.

Urethroplasty should be performed in patients that presented a post-dilatation relapse or a recurrence after an endoscopic urethrotomy for stenoses less than $2 \mathrm{~cm}$ in length. Urethroplasty is also the preferred treatment for patients with long $(\geq 2 \mathrm{~cm})$ bulbar urethral strictures, given the low success rate of direct visual internal urethrotomy (DVIU) or dilation. The surgical reconstruction of the urethral strictures can be carried out using oral mucosal grafts, penile fascia-cutaneous flaps or a combination of these techniques. In literature are reported isolated cases of urethral carcinoma following urethroplasty $(1,2)$. We report our experience in order to increase the scientific evidence.

\section{Case description}

The patient was a 69 years old male who had in the previous year's several endoscopic surgeries for multiple recurrences of urethral stenosis. So in July 2001 an Orandi flap urethroplasty with penile skin was performed. Subsequently in July 2016 the patient presented multiple scrotal and penile urinary fistulae accompanied by urosepsis. Then an abdominal-pelvic MRI identified a multi-segmented abscess of the left inguinal scrotal region and a solid neoplasm involving the urethra and corpora cavernosa in their proximal part. The micturition cystography pointed out also a severe stenosis of the penile urethra, confirmed by an endoscopic exam.

So, a partial penectomy and distal ureterectomy were therefore performed with a perineal urethrostomy.

The operation was accomplished by a median incision of the penile shaft, from the external urethral meatus down to the perineum. The presence of hard tissue, sometimes ulcerated and with the presence of purulent material, which incorporated the urethra up to the bulbar section, made the correct distinction of the surgical planes difficult. In order to better guide the surgical choice, a tissue sample was taken and sent for extemporaneous histological examination which disclosed for "epidermoid carcinoma, affected by moderate interstitial leukocyte exudation, with granulomatous aspects similar to a foreign body".

Given the histological report, it was decided to proceed with total urethrectomy and the forging of a perineal urethral meatus.

In September 2016, the patient undertook an oncological assessment and subsequent cycle of systemic chemotherapy with Cisplatin and 5-FU, which was completed in January 2017. Unfortunately, no significant therapeutic response to systemic therapy was observed. Due to the progression of the disease, the patient began to accuse diffuse pain in the hypogastric and inguinal regions. The MRI restaging demonstrated a neoplastic extension of the disease into the corpora cavernosa up to the bulbs and neighboring regions.

In March 2017 the patient underwent new surgery.

We proceeded with a median longitudinal incision at the level of the pubic symphysis and extended caudally down to the perineal urethral meatus, also invaded by neoplastic tissue. The proximal cavernous bodies appeared rigid and hard, deeply affected by the neoplasia. Even the tissues nearest to the corpora cavernosa bulbs appeared to be affected by the neoplasm.

For this reason we proceeded to a detachment of the 
neoplastic plate from the periosteum of the pubis and its excision up to the bulbar section of the urethral stump and so on to a total penectomy, en-block removal of external genitalia and scrotal sac. Given the extent of the

\section{Figure 1.}

Histology reports: "moderately differentiated spinocellular carcinoma with horn pearl formations of the periurethral tissue and the corpora cavernosa" H\&E stain. 100x.

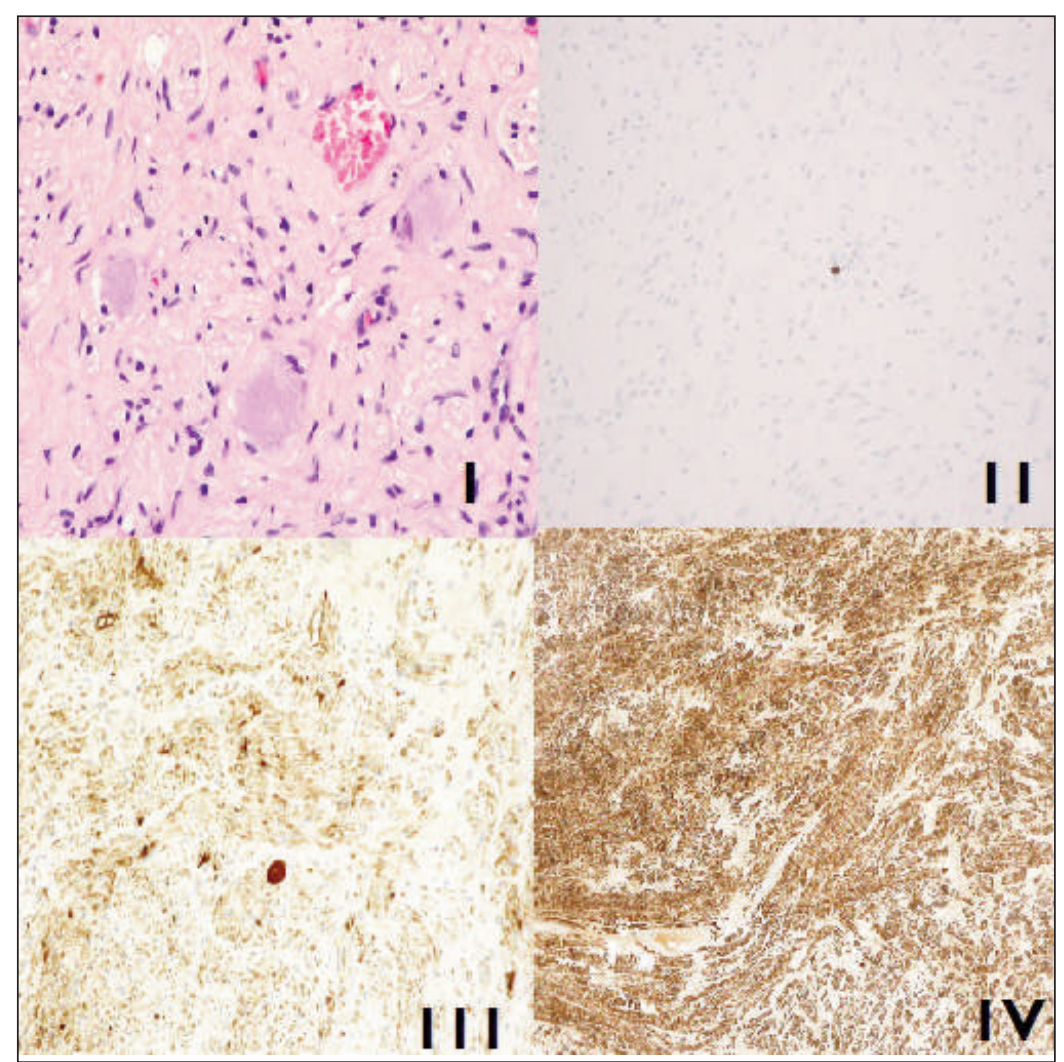

tumor and its degree of infiltration, it was not possible to guarantee oncological radicalness. The intervention was completed by accomplishing a definitive suprapubic urinary derivation. The definitive histological report showed a "moderately differentiated spinocellular carcinoma with horn pearl formations of the periurethral tissue and the corpora cavernosa, focally extended to the testicular albuginea without infiltrating the pulp. The neoplastic involvement includes the tissues of the scrotum up to the dermis". The rapid progression of the disease and the state of clinical impairment of the patient did not allow to consider treatments with an adjuvant intent. The patient died in June 2017

\section{Conclusions}

Although extremely rare, the malignant transformation of the skin flaps used during urethroplasty is possible. Such carcinoma if not early recognized can have harmful consequences as documented. It is therefore important to undertake a long urological follow-up in patients subjected to this kind of urethral surgery.

\section{REFERENCES}

1. Williams G, Ashken MH. Urethral carcinoma following urethroplasty. J R Soc Med. 1980; 73:370-1.

2. Sawczuk I, Acosta R, Grant D, et al. Post urethroplasty squamous cell carcinoma. N Y State J Med. 1986; 86:261-3.

\section{Correspondence}

Danilo Abate, MD

Giuseppe Giusti, MD (Corresponding Author)

giuseppegiusti.med@gmail.com

Nicola Caria, MD (Corresponding Author)

nicolacarial@gmail.com

Giuseppe De Vita, MD

Marco Lucci Chiarissi, MD

Antonello De Lisa, MD

Clinica Urologica, Università degli Studi di Cagliari, Ospedale SS. Trinità

Via Is Mirrionis, Cagliari, Italy 